\title{
Gefährdet Multikulturalität tatsächlich Vertrauen und Solidarität? Eine Replik
}

\section{Einleitung: Kontroversen über Vielfalt und soziale Integration}

Im Juniheft 2011 des Leviathan entwickelte Jürgen Gerhards eine umfassende Kritik einer Förderung von Minderheitensprachen, wie sie im Rahmen internationaler Vereinbarungen zum Minderheitenschutz verfolgt wird. Diese könne sowohl »zu individuellen Nachteilen für die Mitglieder der Minderheitengruppe « als auch "zu negativen Effekten auf der Makroebene « führen. Letztlich könne eine solche Politik dazu beitragen, dass die "soziale(n) Integration der Gesellschaft insgesamt "1 geschwächt werde. Denn Sprachförderung sei in der Regel Teil einer umfassenderen Minderheitenförderung, und diese befestige ethnische und kulturelle Heterogenität. Deren Folge, so die Annahme, seien verringerte soziale Interaktion und reduziertes Vertrauen, Kernelemente von »Sozialkapital « im Putnam'schen Sinne. Die Kontroverse betrifft also nicht allein das Recht auf Erhalt des Galizischen oder Walisischen und damit vielleicht etwas obskure Randthemen internationaler Politik, sondern Grundfragen der Verfassung und Stabilität heterogener Gesellschaften.

Gerhards selbst weist darauf hin, dass die empirischen Befunde zum Zusammenhang von ethnisch-kultureller Heterogenität und Sozialkapital nicht eindeutig und weitere empirische Forschungen wünschenswert seien. In der Tat sind die Thesen von Robert Putnam, auf die Gerhards sich hier stützt, überaus umstritten. Etliche empirische Arbeiten vor allem zu europäischen Kontexten kommen zu abweichenden Ergebnissen. ${ }^{2}$ Dies gilt auch für unsere am Max-Planck-Institut zur Erforschung multiethnischer und multireligiöser Gesellschaften durchgeführte Studie, die erstmals umfassend für kleinräumige Einheiten in Deutschland ${ }^{3}$ Auswirkungen der

1 Gerhards 2011, S. 182-183.

2 Vgl. zu Großbritannien z. B. Laurence 2011; zu den Niederlanden etwa Tolsma et al. 2009, im Überblick van der Meer, Tolsma 2011; Portes, Vickstrom 2011. Die unterschiedlichen Studien sind nicht ohne weiteres vergleichbar, da sowohl die räumlichen Einheiten als auch die Definitionen von Diversität und »Sozialkapital «, »social cohesion « etc. uneinheitlich sind. Als Tendenz lässt sich dennoch feststellen, dass Effekte uneinheitlich sind und ethnische Vielfalt, wenn überhaupt, schwächer wirkt als sozioökonomische Faktoren.

3 Wir gehen davon aus, dass als lebensweltlicher Erfahrungsraum einer Person kleinräumige Kontexte eine wichtige Rolle spielen. Für Deutschland gibt es bislang nur Befunde zu Auswirkungen ethnischer Heterogenität, die sich auf relativ große und in sich heterogene Kontexte beziehen, wie Postleitzahlbezirke (Drever 2008) oder Raumordnungsregionen (Gundelach, Traunmüller 2010). Zum Team der Göttinger »Diversity and Contact «-Studie (DivCon) gehören neben den AutorInnen Dietlind Stolle (McGill University, 
Diversität der Bevölkerungszusammensetzung auf soziale Interaktionen und Indikatoren gesellschaftlicher Integration untersucht. Bis vor kurzem stützte sich die Diskussion wesentlich auf empirische Ergebnisse für Nordamerika. Ob aber diese umstandslos auf europäische Wohlfahrtsstaaten anwendbar sind, ist zweifelhaft. Wie wir im Folgenden belegen werden, sind Szenarien, die eine ethnische Heterogenität der Bevölkerung für die Erosion von Vertrauen und Solidarität verantwortlich machen, zumindest für Deutschland - und wohl auch darüber hinaus - nicht haltbar.

Um dies zu untermauern, werden wir Ergebnisse zu zwei Fragestellungen vorstellen: Zunächst fragen wir, ob es einen Zusammenhang zwischen der Bevölkerungsstruktur (hier ihrer migrationsbedingten Zusammensetzung) des Wohnviertels und der Art, Zahl und Dichte zwischenmenschlicher Beziehungen gibt. Die Desintegrationsthese unterstellt, Menschen in heterogenen Gegenden ${ }^{4}$ lebten »zurückgezogener «. ${ }^{5}$ Ist dies der Fall? Und wie könnte ein solcher Zusammenhang - wenn es ihn denn gibt - funktionieren; welche Mechanismen werden hier angenommen? Unterstellt wird, dass ethnische Heterogenität nicht nur ein Problem in der Interaktion der ethnisch Verschiedenen ist, sondern auch die Interaktion der ethnisch Verwandten einschränkt. Dieses von Putnam vorgetragene Argument knüpft an die in der sozialpsychologischen Diskussion gängige Bedrohungshypothese (threat-hypothesis) an, die davon ausgeht, dass die Anwesenheit Fremder Bedrohungsgefühle auslöst, die wiederum Rückzugstendenzen provozieren. Dagegen spricht sich etwa $\mathrm{Blau}^{6}$ in seiner makrosoziologischen Strukturtheorie dafür aus, dass Heterogenität vor allem im Sinne von Kontaktopportunitäten interpretiert werden sollte. Werden solche Opportunitäten genutzt, so die Kontakttheorie, dann befördern die Kontakte positive Einstellungen zueinander. ${ }^{7}$ Wir fragen zunächst, ob die direkten sozialen Interaktionen durch den Wohngebietskontext beeinflusst werden, ob also unterschiedliche Grade ethnischer Diversität mit mehr oder weniger Interaktion einhergehen.

Zweitens zeigen wir Ergebnisse zur Frage, ob es einen Zusammenhang zwischen der ethnischen Diversität von Wohngebieten und der Einstellung ihrer Bewohner zu anderen Menschen gibt. Letzeres wird häufig mit Hilfe einer Reihe von Standardfragen zu Graden des Vertrauens in die Menschen generell oder in bestimmte Gruppen untersucht. Das so gemessene Vertrauen gilt oft als Grundlage von sozialem Kapital, zivilgesellschaftlichem Engagement und Solidarität. ${ }^{8}$ Die Annahme, dass

Montréal), Miles Hewstone und Katharina Schmid (Oxford University) sowie Steven Vertovec, Jörg Hüttermann (MPI MMG Göttingen) und Thomas Schmitt (jetzt Universität Erlangen).

4 Gerhards übernimmt den Begriff »Community « als »Gemeinschaft «. Wir ziehen es vor, neutraler von der Bevölkerung bestimmter Gebiete zu sprechen.

5 Gerhards 2011, S. 183.

6 Blau 1994, S. $28 \mathrm{ff}$.

7 Vgl. z. B. Hewstone 2009; Wagner et al. 2006.

8 So auch Gerhards 2011, S. 183. 
ethnische Heterogenität das Vertrauen der Bürger in andere Bürger untergräbt, ist Kern des Desintegrationsarguments, wie es auch Gerhards, gestützt auf Putnam ${ }^{9}$, referiert. Tatsächlich werfen die gängigen Indikatoren für Vertrauen bzw. die entsprechenden Daten viele Fragen auf; die Deutung der durchaus relevanten Unterschiede z. B. zwischen Staaten ist keineswegs einfach. ${ }^{10} \mathrm{Ob}$ die gängigen Fragen nach dem Vertrauen der Individuen in andere Menschen tatsächlich die beste Art darstellen, gesellschaftliche Integration (selbst sicherlich keineswegs ein eindeutiger Begriff) zu messen, sei dahingestellt. Alejandro Portes und Erik Vickstrom etwa haben kürzlich dagegen argumentiert, gegenseitiges Vertrauen und Kegelklubs (Putnams Beispiel für Vereine und ein funktionierendes gesellschaftliches Leben) seien zwar ganz schön, aber keine unverzichtbaren Eigenschaften einer tragfähigen Gesellschaft. ${ }^{11}$ Moderne Gesellschaften bräuchten Homogenität und Kommunitarismus nicht, um ihr soziales Leben in einer für Individuen und Kollektive zuträglichen Art zu organisieren. Starke Institutionen seien zentral für deren Bestand. Wir teilen diese Argumentationslinie im Grundsatz. Wenn unsere Studie dennoch - auch im Interesse ihrer Vergleichbarkeit mit anderen Untersuchungen - gängige Fragen nach dem Vertrauen in andere Menschen verwendet, geschieht dies nicht im Sinne eines Putnam'schen Konzepts sozialen Kapitals oder sozialer Integration; vielmehr verstehen wir diese Aussagen als einen Indikator der Einstellungen von Individuen zu ihrer sozialen Umwelt.

\section{Empirische Befunde der Göttinger DivCon-Studie}

Die im Folgenden präsentierten Daten basieren auf einer repräsentativen Umfrage in deutschen Städten. Im Rahmen einer geschichteten Zufallsauswahl wurden 50 Wohnviertel ausgewählt, die sich nach dem Ausländeranteil in der Bevölkerung ${ }^{12}$ und ihrem sozialen Status ${ }^{13}$ unterscheiden. In jedem Stadtgebiet wurden 50 Erwachsene telefonisch befragt, insgesamt also 2.500 Personen. 21 Prozent von ihnen haben einen Migrationshintergrund.

Gibt es einen Einfluss der Bevölkerungsstruktur eines Wohngebiets, hier des Ausländeranteils, auf die persönlichen sozialen Beziehungen seiner BewohnerInnen? Erfasst wurden drei verschiedene Formen bzw. Grade persönlicher Beziehungen: Unterhaltungen im Wohnviertel, der Bekanntenkreis und die engeren Freundschafts- und familiären Beziehungen. Dabei interessierten uns Zahl und Dichte solcher Beziehungen sowie die Interaktionen zwischen Menschen mit und ohne Migra-

9 Putnam 2007.

10 Vgl. Newton 2007; Hartmann, Offe 2001; Bjørnskov 2008.

11 Portes, Vickstrom 2011, S. 472.

12 Es gibt im Moment keine Alternative zur Nutzung der Ausländerdaten in einer derartigen Studie, da Daten zum Migrationshintergrund nicht für kleine Gebiete und alle deutschen Städte verfügbar sind.

13 Hier operationalisiert als Anteil der Arbeitslosen an den 15- bis 64-Jährigen. Auch hier gibt es Datenprobleme für kleine Gebiete. 
tionshintergrund. Betrachtet man zunächst diese letzte Form der »interethnischen « Interaktionen, dann zeigt sich, dass die Eigenschaften der Wohnumgebung durchaus einen Einfluss haben. Insbesondere der wohl eher lockere Kontakt im Wohngebiet zwischen Menschen mit und ohne Migrationshintergrund korreliert mit bestimmten Eigenschaften der Bevölkerungsstruktur: Dort, wo mehr AusländerInnen leben, gibt es auch häufiger solche Kontakte, die wir mit einer Frage nach der Häufigkeit von Unterhaltungen gemessen haben (Abbildung 1). ${ }^{14}$ Das mag banal wirken, widerspricht aber diametral der Desintegrationsthese, die ja behauptet, dass Heterogenität Rückzug befördert. Über alle Wohngebiete hinweg sind solche Kontakte relativ häufig: Im Durchschnitt haben 53 Prozent aller Befragten täglich oder mindestens einmal wöchentlich solche Kontakte, nur 13 Prozent haben sie nie.

\section{Abbildung 1: Intergruppen-Kontakte im Wohnviertel nach Ausländeranteil im Wohnviertel}

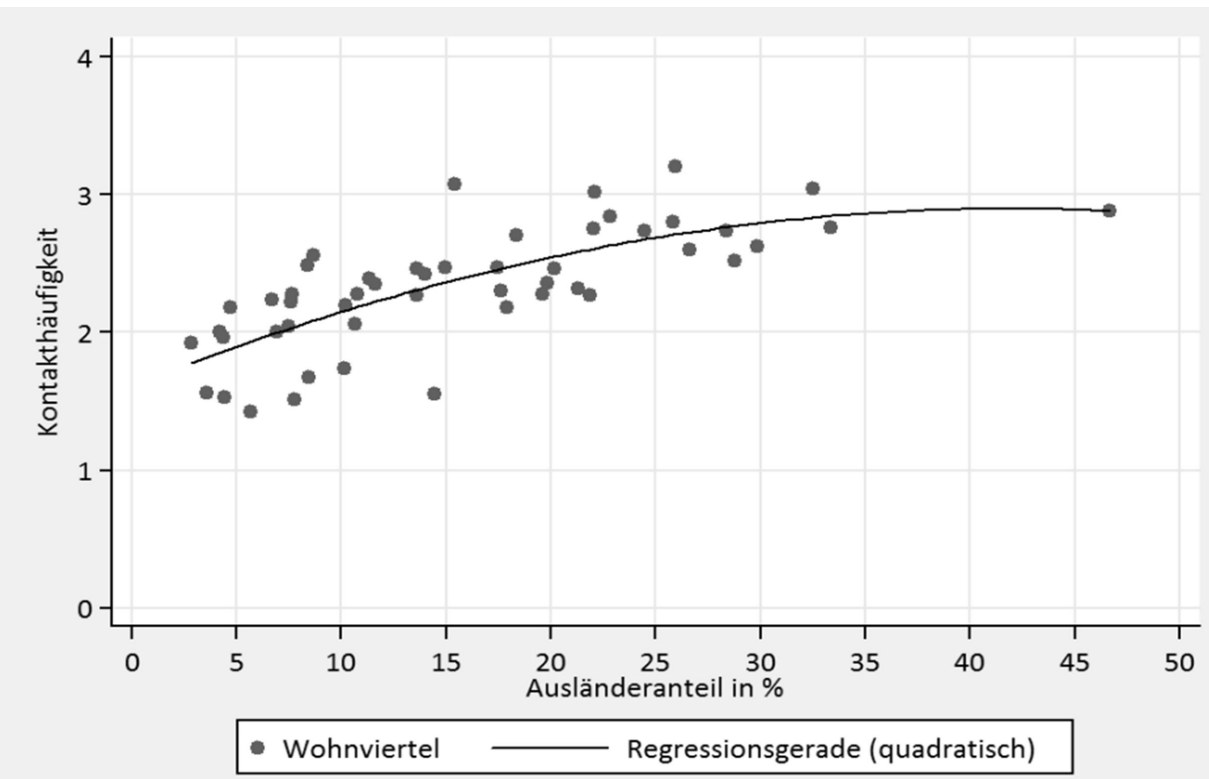

DivCon Survey 2010: $\mathrm{n}=2.490$ in 50 Wohnvierteln

Nun wäre es denkbar, dass Unterhaltungen spannungsreich sind oder als Streit stattfinden. Dies ist aber nicht der Fall. Die Begegnungen werden ganz überwiegend positiv erlebt. Nur eine winzige Gruppe unserer Interviewpartner beschreibt die

14 »Jetzt nochmal eine Frage zu Ihrem Wohnviertel. Wie oft unterhalten Sie sich in Ihrem Wohnviertel mit Menschen, die selbst oder deren Eltern nicht aus Deutschland stammen/ die aus Deutschland stammen? « [an Interviewpartner ohne bzw. mit Migrationshintergrund]. Die Antwortkategorien waren »täglich" (4), »mindestens einmal in der Woche« (3), »mindestens einmal im Monat« (2), »seltener« (1) und »nie« $(0)$. 
gruppenübergreifenden Unterhaltungen im Wohnviertel als » unangenehm « (1 Prozent), eine größere Gruppe (22 Prozent) entzieht sich und antwortet »weder noch ", über drei Viertel aber beschreiben sie als »angenehm «. ${ }^{15}$

Auch allgemeiner finden wir keine Rückzugstendenzen: Die Häufigkeit von Unterhaltungen generell ${ }^{16}$ ist über alle Wohnviertel hinweg sehr hoch. Auch die Größe der Netzwerke, also die Zahl der Bekannten und engeren Freunde, weist keinen Zusammenhang auf mit der Diversität der Bevölkerung im Wohngebiet. ${ }^{17}$ Ähnliches gilt für die Dichte des gesamten persönlichen Netzwerks, also die Frage, ob die Mitglieder des Netzwerks sich auch untereinander kennen. Insgesamt gibt es kein Indiz dafür, dass ethnische Heterogenität sozialen Rückzug und Entfremdung fördert.

Bestätigen sich diese Ergebnisse ebenso bezüglich des Vertrauens der Individuen in andere Menschen? Hier ist das Bild etwas uneinheitlicher, aber auch für den Zusammenhang von Heterogenität und Vertrauen sehen wir keine Bestätigung für Desintegrationsthesen. Wir haben sowohl eine allgemeine Vertrauensfrage ${ }^{18}$ gestellt als auch gezielt nach dem Vertrauen in drei große Gruppen anderer als deutscher Herkunft gefragt, und zwar in Türkeistämmige, Russlanddeutsche (als alltagssprachlichem Äquivalent für Spätaussiedler) und in andere Westeuropäer.

Testet man zunächst bivariat das Verhältnis zwischen dem Ausländeranteil im Gebiet und dem interpersonalen Vertrauen dort lebender Befragter ganz allgemein, dann zeigt sich - wie die Abbildung 2 illustriert - kein Zusammenhang beider Indikatoren. Für die allgemeine Vertrauensfrage ist zudem die Varianz zwischen den Wohnvierteln überaus gering: Die Durchschnittswerte der mit einer Fünfer-Skala gemessenen Antworten ${ }^{19}$ bewegen sich in einem schmalen Korridor zwischen 2,9 und 3,7 um den Gesamtdurchschnitt von 3,2. Ob ein Gebiet einen unteren oder einen oberen Wert aufweist, scheint - nach dieser ersten Analyse - mit der Zahl der dort lebenden AusländerInnen nichts zu tun zu haben.

15 Zwischen den Befragten mit bzw. ohne Migrationshintergrund gibt es hier keine großen Unterschiede. Zwar äußern sich MigrantInnen positiver als NichtmigrantInnen, in einem Regressionsmodell, das zahlreiche Drittvariablen kontrolliert, findet sich aber kein signifikanter Zusammenhang.

16 Neben der oben zitierten Frage nach den »interethnischen « Kontakten haben wir auch gefragt: "Wie oft unterhalten Sie sich mit alteingesessenen Deutschen? « (Interviewer: Noch einmal zur Erinnerung: Wir meinen hier Deutsche, die nicht eingewandert sind und deren Eltern auch schon Deutsche waren.)

17 Der Anteil der MigrantInnen in solchen Netzwerken der NichtmigrantInnen wird anders als die Häufigkeit von Unterhaltungen zwischen Mitgliedern beider Gruppen im Wohngebiet - nicht durch die ethnische Heterogenität der Wohnviertel beeinflusst. Dies liegt wohl daran, dass Bekannte und Freunde nicht vorwiegend im Wohngebiet gefunden werden.

18 Die im Fragebogen verwendete Formulierung lautet: »Ganz allgemein gesprochen: Glauben Sie, dass man den meisten Menschen vertrauen kann oder dass man im Umgang mit ihnen nicht vorsichtig genug sein kann? «

19 Die Antwort 1 entspricht der Meinung »Man kann nicht vorsichtig genug sein «, die 5 steht für »Man kann den meisten Menschen vertrauen«, 2 bis 4 erlauben Abstufungen. 


\section{Abbildung 2: Allgemeines Vertrauen nach Ausländeranteil im Wohnviertel}

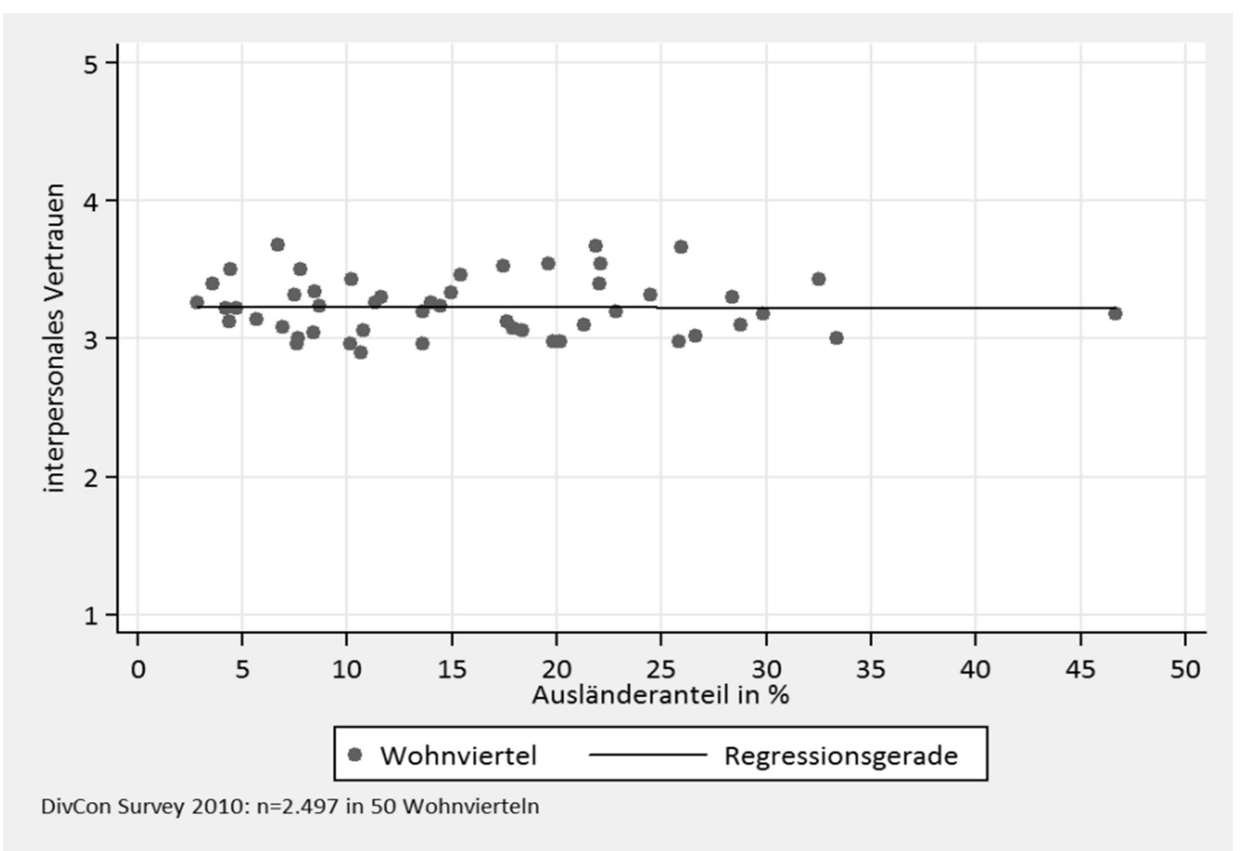

Ähnlich ist das Bild für das Vertrauen in »andere " (hier als Index aus dem Vertrauen in die drei oben genannten Gruppen). Auch hier zeigt die bivariate Analyse keinen Zusammenhang. Dies mag überraschen, denn es wäre ja durchaus plausibel, anzunehmen, dass in einem Gebiet, in dem die Menschen sehr verschieden sind, das gegenseitige Vertrauen in "andere " geringer ist, weil man glaubt, sich weniger zu kennen. Solche Gebiete sind zudem häufig Regionen, in denen die Bewohnerschaft sich schneller verändert als anderswo, wo auch Arbeitslosigkeit und Armut stärker verbreitet sind - Faktoren, deren negative Korrelation mit dem Vertrauen bekannt ist. Umgekehrt könnte man aber auch vermuten, dass durch die Koexistenz Vertrauen entsteht. Unsere Analysen zeigen aber weder einen positiven noch einen negativen Zusammenhang; beide Vermutungen werden nicht bestätigt. ${ }^{20}$

Isoliert man in einer Serie von Regressionsmodellen einzelne Einfluss nehmende Faktoren, dann zeigt sich auch hier die Diversität im Wohngebiet als irrelevant gerade für das Vertrauen in die »Anderen « (outgroup trust), während es einen leicht negativen Einfluss höherer Ausländeranteile auf das allgemeine Vertrauen gibt. Dieser Einfluss ist aber überaus gering. Ist der Ausländeranteil um einen Prozentpunkt höher, bewirkt dies einen um 0,006 niedrigeren Wert auf der Vertrauensskala. Warum die Präsenz von MigrantInnen Auswirkungen auf das Vertrauen in Men-

20 Denkbar ist auch, dass entsprechende gegensätzliche Effekte existieren, sich aber gegenseitig aufwiegen. 
schen allgemein - nicht aber speziell in große Migrantengruppen haben soll, gehört zu den Rätseln, die uns die Vertrauensfrage aufgibt. In jedem Fall aber ist der Diversitätseffekt auf Vertrauen - wenn überhaupt - nur schwach ausgeprägt.

Hinzu kommt, dass in der Realität die statistisch isolierbaren, leicht negativen Wirkungen von Diversität auf das allgemeine Vertrauen nicht entsprechend auftreten, da es in Wohngebieten mit hohem Ausländeranteil auch zu häufigen sozialen Interaktionen kommt. Solche Begegnungen wiederum, wie wir sie als »Unterhaltungen im Wohnviertel « gemessen haben, wirken offenbar positiv auf beide Typen von Vertrauen. Im Ergebnis ist in Gebieten mit höheren Ausländeranteilen das durchschnittliche Vertrauen in andere Menschen ähnlich hoch oder niedrig wie in Gebieten, die relativ homogen deutsch sind.

\section{Bilanz}

Insgesamt sprechen unsere Ergebnisse dafür, dass es die gelegentlich vermuteten negativen Wirkungen hoher Migrantenanteile auf soziale Interaktionen und gegenseitiges Vertrauen in Deutschland nicht gibt. Vielmehr scheint die Diversität der Bevölkerung im Wohngebiet in mancher Hinsicht irrelevant zu sein und auf lockere Interaktionen zwischen Menschen mit und ohne Migrationsgeschichte positiv zu wirken. Auf der Basis von SOEP-Daten kam Anita Drever vor einigen Jahren zu ähnlichen Ergebnissen: »Germans living within the country's ethnic neighborhoods appear to have numbers of close friends and levels of social trust equal to their compatriots outside these areas «.21 Möglicherweise hängt die Art und Weise, wie ethnische bzw. migrationsbedingte Vielfalt verarbeitet wird, mit weiteren Eigenschaften des auch nationalen Kontextes zusammen. Vielleicht weichen unsere Ergebnisse für Deutschland von denen einiger auf die USA bezogener Studien ab, weil bei einem relativ geringen Ausmaß räumlicher Segregation, stärkeren wohfahrtsstaatlichen Strukturen, geringeren Unterschieden zwischen den Lebensbedingungen in unterschiedlichen Stadtvierteln und ohne einen durch die Geschichte der Sklaverei bestimmten Gegensatz von Schwarz und Weiß Diversität anders auf das soziale Leben wirkt. Vergleichende Studien sollten hier weitere Klärung bringen. Unsere Ergebnisse für die Situation in Deutschland widerlegen jedenfalls Befürchtungen, wonach die durch Migrationsprozesse gewachsene Heterogenität mit einem "Nachlassen des Grades der sozialen Integration der Gesellschaft insgesamt «22 einhergeht. Theoretisch wirft die unterstellte Wirkungskette von gemischten Wohngebieten über Bekanntschaftsnetzwerke und die Sammelkategorie Vertrauen hin zur Integration der Gesellschaft insgesamt ohnehin viele Fragen auf. Heterogenität ist ein Charakteristikum moderner Gesellschaften, mit dem sie durchaus zurechtkommen können. Ein Plädoyer für sprachliche und andere Assimilation, wie es ja die

21 Drever 2008, S. 187. Esser kam in den 1980er Jahren nach Studien in Duisburg zu dem Ergebnis, dass makrostrukturelle Kontexte inklusive unterschiedlicher Ausländeranteile im Wohngebiet wenig Einfluss auf Ablehnung und Intergruppenkontakte haben; vgl. Esser 1986, S. 49.

22 Gerhards 2011, S. 183. 
Konsequenz von Gerhards' Argumentation ist, ist unter Hinweis auf einen bedrohten sozialen Zusammenhalt nicht plausibel begründbar.

\section{Literatur}

Bjørnskov, Christian 2008. "Social trust and fractionalization: a possible reinterpretation «, in European Sociological Review 24, S. 271-283.

Blau, Peter M. 1994. Structural context of opportunities. Chicago, London: University of Chicago Press.

Drever, Anita I. 2008. »Germans in Germany's ethnic neighborhoods «, in Schmollers Jahrbuch. Zeitschrift für Wirtschafts- und Sozialwissenschaften 128, 1, S. 175-190.

Esser, Hartmut 1986. »Social context and interethnic relations. The case of migrant workers in West German urban areas", in European Sociological Review 2, S. 30-51.

Gerhards, Jürgen 2011. »Der Kult der Minderheitensprachen «, in Leviathan 39, 2, S. 165-186.

Gundelach, Birte; Traunmüller, Richard 2010. »Kulturelle Diversität und sozialer Zusammenhalt. Eine Mehrebenenanalyse zum Einfluss multikultureller Kontexte auf das Sozialkapital in den deutschen Regionen ", in Vergleichende subnationale Analysen für Deutschland, hrsg. v. Freitag, Markus; Vatter, Adrian, S. 315-344. Berlin: LIT Verlag.

Hartmann, Martin; Offe, Claus 2001. Vertrauen. Die Grundlage des sozialen Zusammenhalts. Frankfurt a. M., New York: Campus.

Hewstone, Miles 2009. Living apart, living together? The role of intergroup contact in social integration. Proceedings of the British Academy 162, S. 243-300.

Laurence, James 2011. »The effect of ethnic diversity and community disadvantage on social cohesion: a multi-level analysis of social capital and interethnic relations in UK communities ", in European Sociological Review 27, 1, S. 70-89.

Meer, Tom van der; Tolsma, Jochem 2011. Ethnic diversity and its supposed detrimental effect on social cohesion; when theory fails (unveröffentlichtes Manuskript).

Newton, Kenneth 2007. "Social and political trust ", in The Oxford handbook of political behaviour, hrsg. v. Dalton, Russell J.; Klingemann, Hans-Dieter, S. 342-360. Oxford: Oxford University Press.

Portes, Alejandro; Vickstrom, Eric 2011. "Diversity, social capital, and cohesion ", in Annual Review of Sociology 37, S. 461-479.

Putnam, Robert D. 2007. »E pluribus unum: diversity and community in the twenty-first century. The 2006 Johan Skytte Prize Lecture", in Scandinavian Political Studies 30, 2, S. 137-174.

Tolsma, Jochem; van der Meer, Tom; Gesthuizen, Maurice 2009. »The impact of neighbourhood and municipality characteristics on social cohesion in the Netherlands ", in Acta Politica 44, 3, S. 286-313.

Wagner, Ulrich et al. 2006. »Prejudice and minority proportion: contact instead of threat effects «, in Social Psychology Quarterly 69, 4, S. 380-390. 
Zusammenfassung: Der Artikel wendet sich gegen Positionen in der wissenschaftlichen Diskussion, die in der migrationsbedingten Diversität eine Ursache negativer Entwicklungen der sozialen Integration der Gesellschaft sehen. Gestützt auf umfangreiche empirische Daten des Göttinger »Diversity and Contact«-Projekts, wird gezeigt, dass in deutschen Städten hohe Anteile von AusländerInnen an der Bevölkerung des Wohnviertels keineswegs mit verminderten sozialen Interaktionen einhergehen. Zweitens wird gezeigt, dass auch die verschiedentlich unterstellte Abnahme des interpersonalen Vertrauens infolge erhöhter Diversität nicht nachweisbar ist. Während die migrationsbezogene Diversität der Wohngebiete für das Vertrauen unter den Menschen relativ irrelevant ist, gibt es in diverseren Gebieten mehr Interaktionen zwischen BewohnerInnen ohne bzw. mit Migrationshintergrund.

Stichworte: ethnische Diversität, Vertrauen, soziale Interaktionen, Desintegrationsthesen, Intergruppenkontakte, Wohnvierteleffekte

\section{Is the multiculturality of neighbourhoods endangering trust and solidarity? A reply to Jürgen Gerhards}

Summary: The article contradicts positions in the academic debate that see migration-related diversity as a negative influence on the social integration of societies. Based on the empirical data of the Göttingen «Diversity and Contact «-study, the authors show that in Germany high shares of foreigners among the inhabitants of urban neighbourhoods do not go along with reduced social interaction. Further, the assumption that interpersonal trust diminishes in response to high diversity is not supported by the German data. While migration-related diversity and trust seem largely unrelated, contact between residents without and with migration backgrounds is more frequent in more diverse areas.

Keywords: ethnic diversity, trust, social interactions, disintegration theses, intergroup-contact, neighbourhood effects

\section{AutorInnen}

PD Dr. Sören Petermann

Max-Planck-Institut zur Erforschung multireligiöser und multiethnischer Gesellschaften

Hermann-Föge-Weg 11

D-37073 Göttingen

petermann@mmg.mpg.de

Prof. Dr. Karen Schönwälder

Max-Planck-Institut zur Erforschung multireligiöser und multiethnischer Gesellschaften

Hermann-Föge-Weg 11

D-37073 Göttingen

schoenwaelder@mmg.mpg.de 